\title{
Fingerprint Classification Using a Feedback-Based Line Detector
}

\author{
Shesha Shah and P. S. Sastry, Senior Member, IEEE
}

\begin{abstract}
We present a fingerprint classification algorithm in this paper. This algorithm classifies a fingerprint image into one of the five classes: Arch, Left loop, Right loop, Whorl, and Tented arch. We use a new low-dimensional feature vector obtained from the output of a novel oriented line detector presented here. Our line detector is a co-operative dynamical system that gives oriented lines and preserves multiple orientations at points where differently oriented lines meet. Our feature extraction process is based on characterizing the distribution of orientations around the fingerprint. We discuss three different classifiers: support vector machines, nearest-neighbor classifier, and neural network classifier. We present results obtained on a National Institute of Standards and Technology (NIST) fingerprint database and compare with other published results on NIST databases. All our classifiers perform equally well, and this suggests that our novel line detection and feature extraction process indeed captures all the crucial information needed for classification in this problem.
\end{abstract}

Index Terms-Biometrics, feedback-based line detection, fingerprint classification, neural network classifiers, support vector machines.

\section{INTRODUCTION}

$\mathbf{F}$ INGERPRINT classification is an important subproblem for fingerprint recognition/matching with large databases. Multilevel fingerprint recognition with fingerprint classification at first level improves the computational complexity substantially by reducing the number of candidate matches to be considered.

Fingerprints are the characteristic structure of flow lines of ridges and furrows that are present on the skin on one's finger. It is well known that fingerprints are unique and are well suited for person identification [1], [2]. Automated systems for fingerprint matching are receiving a great deal of attention over the last decade and many commercial systems are available today. For automatic person identification based on fingerprints, the characteristic features obtained from the test fingerprint are to be matched against those in the system database.

When fingerprint image database is large (e.g., in applications relating to law enforcement), it is desirable to have a systematic partitioning to reduce time and complexity involved in fingerprint recognition. Fingerprint classification is one way of establishing such a partitioning. As established in literature [3], fingerprints are classified in six classes: Right loop, Left loop, Arch, Tented arch, Scar, and Whorl. The categorization of fin-

Manuscript received May 13, 2002; revised October 10, 2002. This paper was recommended by Associate Editor V. Govindaraju

The authors are with the Department of Electrical Engineering, Indian Institute of Science, Bangalore-560 012, India (e-mail: shesha@ee.iisc.ernet.in sastry@ee.iisc.ernet.in).

Digital Object Identifier 10.1109/TSMCB.2002.806486 gerprints into arch, etc., is based on the shape of the orientation field of ridge lines in the fingerprint image. Hence, most methods for fingerprint classification start with some kind of edge detection and post processing to obtain the ridge lines. The local orientation of the ridge lines is also detected. One then uses some global features of the flow information and ridge line shape to effect classification [4]-[10]. Karu and Jain [6] first determine the singularity region of the orientation flow field (called core and delta points) from the ridge line map to get a coarse classification. This is followed by a fine classification based on ridge line tracking around singularities. They further extended it to a two-stage classifier [8]. Fitz and Green [5] obtain Hexagonal fast Fourier transform (FFT) of the spatial orientation flow field. The frequency domain features so obtained are then used for classification through nearest-neighbor method. Chong et al. [7] fit a B-spline representation for the ridge lines and then employ a global geometric shape analysis of the ridge lines. Watson et al. in [9] used two types of Fourier transform based filters to enhance fingerprint images and used neural network for fingerprint classification. In [10], Blue et al. used the Karhunen-Loève (K-L) transform of the ridge directions to generate the input feature set, and they have presented results with statistical and neural network classifiers for fingerprint classification.

In our method of fingerprint classification, we use feature vectors obtained from the output of a novel oriented line detector. Our line detector gives the ridge lines along with the orientation information at each point. A special feature of the line detector is that it retains more than one orientation at points where there may be evidence for differently oriented lines meeting or crossing each other. These multiple orientation points are very useful for subsequent fingerprint analysis, because they contain most of the so-called minutiae. ${ }^{1}$ Our line detector is a distributed cooperative dynamic network which iterates a few times to stabilize detected lines. The line detection is based on assessing support for different orientations in local neighborhoods. We employ a novel feedback mechanism to properly segregate evidence for different orientations at each point. This is what is responsible for the ability of the line detector to retain more than one orientation at a point if "sufficient independent evidence" exist for them. This line detection is a specialized and tuned (for fingerprints) version of a general framework for line detection [11], [12]. Our feature vector is based on dividing the line detected image into zones and clustering the distribution of orientations in each zone.

We implemented three different classification strategies. The first classifier is based on support vector machines (SVMs).

${ }^{1}$ Ridge line bifurcations or terminations are called minutiae 
SVMs represent a theoretically elegant and computationally efficient method of designing classifiers for a two-class pattern recognition problem [13], [14]. We used the sequential mimimal optimization (SMO) algorithm [15] to learn our SVMs. Since SVMs are meant for two-class classification and we need to classify a fingerprint into one of five classes, we used five SVMs organized as a hierarchical classifier. Our second method employs the nearest-neighbor classifier with Euclidean distance between feature vectors. We also show that we can use the support vectors obtained from an SVM method as the prototypes in the nearest-neighbor classifier to improve its accuracy and efficiency. Our third classification strategy employs feedforward neural networks trained with the backpropogation algorithm. We employ five neural networks, each trained as a two-class classifier, organized in a hierarchy.

We present results obtained with each of these classification methods on a National Institute of Standards and Technology (NIST) database of fingerprint images and compare our results with other published results on NIST databases. It is seen that all our classifiers perform very well on this problem. This strongly suggests that our novel line detection and feature extraction method captures well the information needed for fingerprint classification.

The rest of the paper is organized as follows. Section II describes our classification system. Section II-A discusses the line detector, and the feature extraction process is explained in Section II-B. We explain the details of our classifiers in Section II-C. We present results obtained with our classifiers on NIST fingerprint image database [3] in Section III. Our line detector gives good ridge lines along with orientation at all points. In addition, it also flags all points where multiple orientations are possible. Hence, we believe that the line detector should be useful for fingerprint identification/recognition as well. We briefly discuss this aspect of our work and conclude the paper in Section IV.

\section{Fingerprint ClassificATION Algorithm}

Input to our fingerprint classification system is a gray-scale image. Then using our line detector we get the ridge lines along with orientations. A feature vector characterizing distribution of orientations is then extracted. This is followed by classification.

Our fingerprint classification algorithm has three main parts: line detection, feature extraction, and classification. The input to the system is a 256 level gray-scale image as an array of dimension $X \times Y$. Generally, most of the fingerprint image consists of closely spaced light and dark bands corresponding to the ridges and furrows on the finger. For a good fingerprint image, one would expect the intensity histogram to have a strong bimodal structure. Hence, we binarize the image using a threshold, which is calculated as follows. We get the histogram of the gray-scale image and detect two highest peaks in histogram: one in gray-scale range $[0,127]$ and the other in $[128,255]$. The threshold is fixed as the midpoint between these two gray values. This method of binarizing works very well with NIST fingerprint database. In the following subsections, we give details of line detection, feature extraction, and classification methods.

\section{A. Line Detection Algorithm}

The input to the line detector is the binarized image $I(i, j)$. The line detector computes two arrays: $S(i, j, k)$ and $L(i, j, k)$. The value of $S(i, j, k)$ indicates the strength of an edge with orientation $k$ at pixel location $(i, j)$, while the value of $L(i, j, k)$ indicates the strength of a line with orientation $k$ at pixel $(i, j)$. (The reason for maintaining two arrays-one for edge and one for line-becomes evident later on). Essentially, $S(i, j, k)$ is computed using some simple oriented edge masks, while $L(i, j, k)$ is computed as a sum of $S(m, n, k)$ for all $m, n$ in a neighborhood around $(i, j)$. We consider eight orientations separated by $\pi / 8 \mathrm{rad}$, and thus, $k \in\{0, \ldots, 7\}$. Hence, if image is of size $X \times Y$, then the arrays $S$ and $L$ are of dimension $X \times Y \times 8$. The specific equations for $S(i, j, k)$ and $L(i, j, k)$ are explained below.

The novel feature of our line detection is incorporation of a feedback mechanism and iterative computation of $S(i, j, k)$ and $L(i, j, k)$ using the feedback. The feedback mechanism is intended to segregate the evidences for different oriented lines at each point in the image. Operationally, the feedback signal, called $O I^{k}(i, j)$ in the equation below, decides which pixels can contribute to the computation of $S(i, j, k)$ at a specific location $(i, j)$.

The strength of edge at $(i, j)$ in orientation $k, S(i, j, k)$ is computed by

$$
\begin{aligned}
S(i, j, k) & =\phi\left(\sum_{n, m=-M}^{M} \tilde{S}^{k}(i, j, m, n)-\gamma\right) \\
& \text { where } \\
\tilde{S}^{k}(i, j, m, n) & =I(i+m, j+n) E^{k}(m, n) O I^{k}(i+m, j+n) \\
\phi(x) & =x \text { if } x>0 \text { and } \phi(x)=0, \text { otherwise. }
\end{aligned}
$$

Here $E^{k}(m, n)$ is an $(2 M+1) \times(2 M+1)$ bipolar edge mask $^{2}$ for orientation $k$. We take $M=3$. The edge masks for a few orientations are shown in Fig. 1. As can be seen, these are simple oriented edge masks for binary images. The $O I^{k}(i+m, j+n)$ in (1) is a binary feedback signal which is computed using (3) below and $\gamma$ is a threshold. For the moment, if we assume $O I^{k}(i, j)=1 \forall i, j, k$, then (1) represents an edge detector with mask $E^{k}(m, n)$, and the output of computation with the masks is thresholded at $\gamma$. Since the feedback signal $O I$ is binary, it would determine which all pixels $(i+m, j+n)$ should be considered while computing edge strength at $(i, j)$.

The strength of line at $(i, j)$ in orientation $k, L(i, j, k)$ is computed as a sum of $S(i, j, k)$ in a neighborhood around $(i, j)$ :

$$
L(i, j, k)=\sum_{n=-M^{\prime}}^{M^{\prime}} \sum_{m=-M^{\prime}}^{M^{\prime}} S(i+m, j+n, k) .
$$

We sum up the edge strength in a neighborhood of size $\left(2 M^{\prime}+1\right) \times\left(2 M^{\prime}+1\right)$ to obtain the line strength. We have used $M^{\prime}=3$.

The feedback signal $O I^{k}(m, n)$ is computed as follows. Let $k_{m}(i, j)$ be the orientation with highest line strength at $(i, j)$ (at the current iteration). That is

$$
k_{m}(i, j)=\arg \max _{k} L(i, j, k) .
$$

${ }^{2}$ Each element of the array $E^{k}(m, n)$ is either +1 or -1 
$\left[\begin{array}{ccccccc}-1 & -1 & -1 & -1 & -1 & -1 & -1 \\ -1 & -1 & -1 & -1 & -1 & -1 & -1 \\ 1 & 1 & 1 & 1 & 1 & 1 & 1 \\ 1 & 1 & 1 & 1 & 1 & 1 & 1 \\ 1 & 1 & 1 & 1 & 1 & 1 & -1 \\ -1 & -1 & -1 & -1 & -1 & -1 & -1 \\ -1 & -1 & -1 & -1 & -1 & -1 & -1\end{array}\right]$

(a)

$$
\left[\begin{array}{lllllll}
-1 & -1 & 1 & 1 & 1 & -1 & -1 \\
-1 & -1 & 1 & 1 & 1 & -1 & -1 \\
-1 & -1 & 1 & 1 & 1 & -1 & -1 \\
-1 & -1 & 1 & 1 & 1 & -1 & -1 \\
-1 & -1 & 1 & 1 & 1 & -1 & -1 \\
-1 & -1 & 1 & 1 & 1 & -1 & -1 \\
-1 & -1 & 1 & 1 & 1 & -1 & -1
\end{array}\right]
$$

(b)

$$
\left[\begin{array}{ccccccc}
-1 & 1 & 1 & 1 & -1 & -1 & -1 \\
-1 & 1 & 1 & 1 & -1 & -1 & -1 \\
-1 & 1 & 1 & 1 & -1 & -1 & -1 \\
-1 & -1 & 1 & 1 & 1 & -1 & -1 \\
-1 & -1 & -1 & 1 & 1 & 1 & -1 \\
-1 & -1 & -1 & 1 & 1 & 1 & -1 \\
-1 & -1 & -1 & 1 & 1 & 1 & -1
\end{array}\right]
$$

(c)

$$
\left[\begin{array}{ccccccc}
1 & 1 & -1 & -1 & -1 & -1 & -1 \\
1 & 1 & 1 & -1 & -1 & -1 & -1 \\
-1 & 1 & 1 & 1 & -1 & -1 & -1 \\
-1 & 1 & 1 & 1 & 1 & 1 & -1 \\
-1 & -1 & -1 & 1 & 1 & 1 & -1 \\
-1 & -1 & -1 & -1 & 1 & 1 & 1 \\
-1 & -1 & -1 & -1 & -1 & 1 & 1
\end{array}\right]
$$

Fig. 1. Bipolar oriented edge masks in direction $k$, where (a) $k=0$ for angle 0 , (b) $k=4$ for angle $4 \pi / 8 \mathrm{rad}$, (c) $k=5$ for angle $5 \pi / 8 \mathrm{rad}$, (d) $k=6$ for angle $6 \pi / 8 \mathrm{rad}$.

Now, $O I^{k}(i, j)$ is given by

$$
\begin{aligned}
& \operatorname{if}\left(L\left(i, j, k_{m}(i, j)\right)-\frac{1}{8} \sum_{s=0}^{7} L(i, j, s)\right)>\tau \\
& \text { then }\{ \\
& O I^{l}(i, j)=1, \text { if } l=k_{m}(i, j) \\
& =0, \forall l \neq k_{m}(i, j) \\
& \text { \} } \\
& \text { else } O I^{l}(i, j)=1, \forall l
\end{aligned}
$$

where $\tau$ is a threshold.
Thus, at a given $(i, j)$, if line strength of the strongest orientation is greater than the average line strength by at least $\tau$, then $O I^{k_{m}(i, j)}(i, j)=1$, and $O I^{l}(i, j)=0$ for all other $l$. Otherwise, $O I^{l}(i, j)=1, \forall l$.

Our line detector iterates over (1)-(3). To start with, we set $O I^{k}(i, j)=1 \forall i, j, k$. After that, we compute $S(i, j, k)$, $L(i, j, k)$, and $O I^{k}(i, j)$ iteratively untl all $S(i, j, k)$ stabilize. In our simulations, the edge strengths stabilize in about three to five iterations. The final output of the line detector is the binary image obtained by thresholding $S(i, j, k)$ at zero.

Since we start with $O I^{k}(i, j)=1 \forall i, j, k$, our first iteration of computing $S(i, j, k)$ is like any other standard edge detector with oriented edge masks. However, during the subsequent iterations, the $O I^{k}(i+m, j+n)$ term in (1) helps to segregate evidences for different oriented lines at a point. Consider a point $(i+m, j+n)$, which is in a $(2 M+1) \times(2 M+1)$ neighborhood of $(i, j)$. This would contribute to the sum in (1) for computing $S(i, j, k)$ only if $O I^{k}(i+m, j+n)=1$. That would be so only if $k$ is the "dominant" line orientation at $(i+m, j+n)$ during the previous iteration or if there is currently no dominant orientation at $(i+m, j+n)$. In the absence of feedback, all pixels $(i+m, j+n)$ in the $(2 M+1) \times(2 M+1)$ neighborhood around $(i, j)$ would contribute to $S(i, j, k)$ for all $k=0, \ldots, 7$. However due to feedback, each of these points would essentially contribute to $S(i, j, k)$ for only one value of $k$. This type of evidence segregation helps in getting better edge orientations. Another interesting aspect of this mechanism is that it can result in more than one edge orientation being indicated at a point if differently oriented lines cross at a point. Detection of such points is useful for subsequent analysis of fingerprint images, such as detection of minutiae.

The line detection algorithm presented here is a specialized version of a general line detector incorporating feedback, which is described in [11] and [12]. A more comprehensive discussion on the role played by feedback and its importance for low-level vision can be found in [11]. The line detector described in [12] uses differentiated oblong Gaussian as edge masks and has two levels of feedback. The algorithm presented here is a new simplified version fine tuned for fingerprint application.

There are only two parameters in this algorithm (apart from mask sizes $M$ and $\left.M^{\prime}\right)$ : $\gamma$ in computing $S(i, j, k)$ and $\tau$ for computing $O I^{k}(i, j)$. The algorithm is fairly robust with respect to them. We have used $\gamma=0.0$ and $\tau=35$ in simulations.

As mentioned earlier, the output of line detection is a binary image obtained by thresholding $S(i, j, k)$ at zero. For subsequent processing, we thinned the line image using a standard thinning algorithm [16]. Fig. 2 shows two fingerprint images from the NIST database and the (thinned) line output of our line detector at iteration 5. The orientation information is coded as grey value in Fig. 2. As can be seen, we get clean ridge lines with orientations. In addition, our line output has coherent orientations. Fig. 3 shows results obtained on fingerprints collected locally. These fingerprint images are noisy and have some breaks (or cracks) which are mostly due to dust and minor cuts on the finger. In spite of such breaks and noise, our line detector gets 

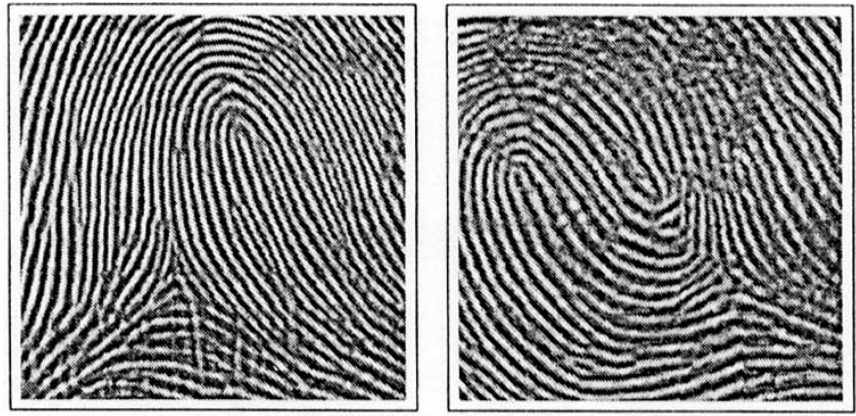

(a)
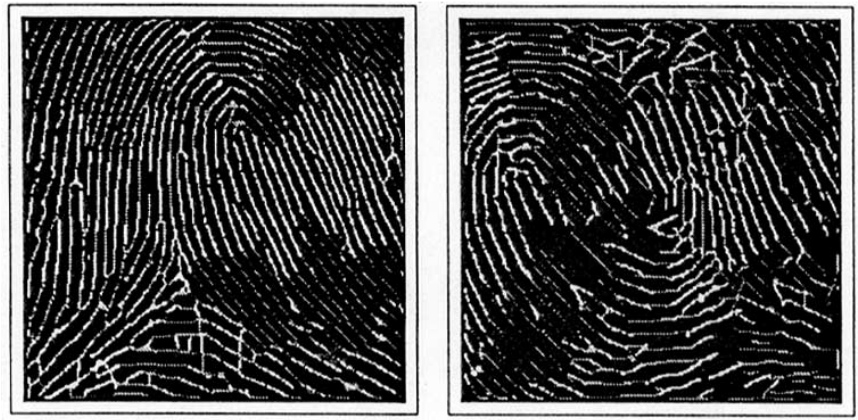

(b)

Fig. 2. Fingerprint images from NIST database. (a) Original images. (b) Line outputs at iteration 5 with orientation shown in different gray level.

clean ridge lines as seen from Fig. 3(b). (Here, we do not show the orientation information.) One of the special features due to our feedback mechanism for evidence segregation is that we automatically signal all the multiple orientations present at a point due to different lines meeting or crossing each other. In Fig. 3(c), we show the points where our line detector signals presence of multiple orientations. In a fingerprint image, the points where ridge lines join or branch out are called minutiae. It is widely believed that the relative locations of and orientations at minutiae contain almost all the information needed for matching fingerprints. Thus, detection of the set of points with multiple orientations (which is mostly a super set of minutiae points) is a very useful bye-product of our line detector. Since we are not considering the fingerprint matching problem here, we do not discuss this point any further.

In Fig. 4, we compare our oriented line output with output from Canny's algorithm [17] and the logical/linear operator [18]. Canny's algorithm takes around 0.15 s/image, and the logical/linear operator take around $15.2 \mathrm{~s} / \mathrm{image}$, whereas our algorithm takes 3.2 s/image. Even though Canny's results are much faster, we do not get coherent oriented lines. We can also see that cracks in fingerprint image are clearly visible in Fig. 4 on both the Canny and logical/linear operator line outputs, which would be noise for any fingerprint classifier. However, as shown in Fig. 3, we are able to make up for the same.

The performance improvement of our line detector is essentially due to the feedback driven dynamics. It is mainly due to our feedback that we are able to fill in the ridge lines through
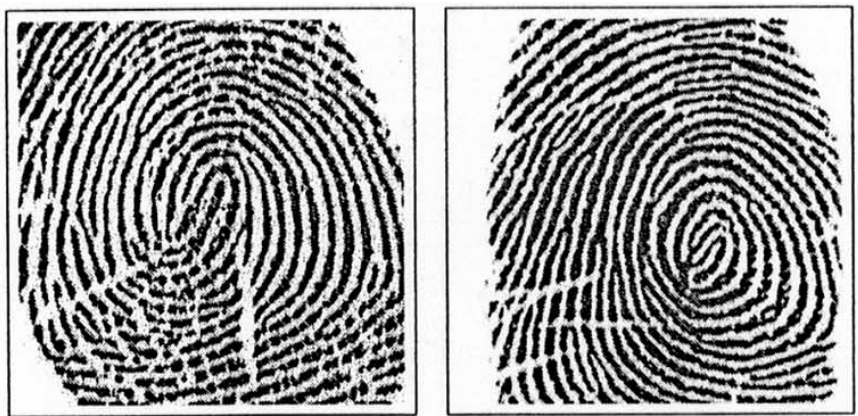

(a)
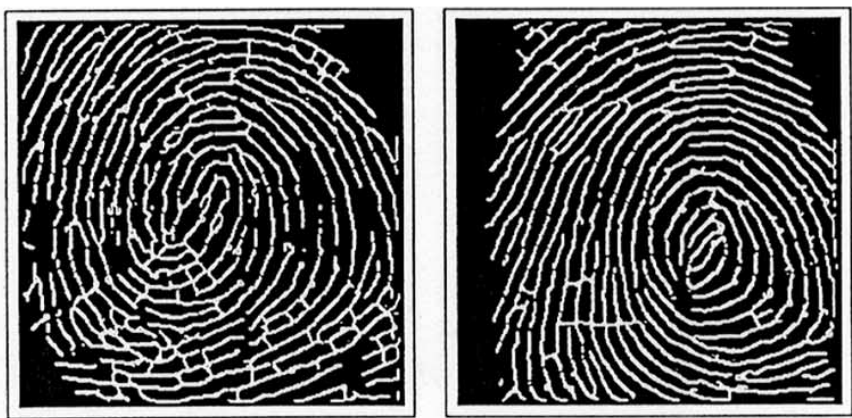

(b)
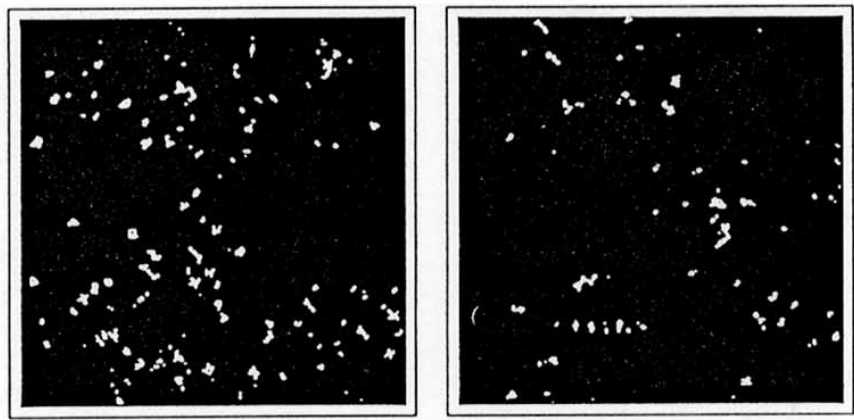

(c)

Fig. 3. Fingerprints from a local database. (a) Original images. (b) Line outputs. (c) Multiple orientation points.

breaks and cracks as well as avoid detecting lines that are artifacts of such breaks. This is illustrated in Fig. 5. Fig. 5(a) shows the original gray fingerprint image, and Fig. 5(b) shows the binary image obtained through our automatic thresholding scheme. Fig. 5(c) shows output of line detector at iteration 1, which would be same as that by any oriented edge detector using very simple bipolar masks. (Recall that for the first iteration, we have $\left.O I^{k}(i, j)=1, \forall i, j, k\right)$. Fig. 5(d)-(i) shows output at different iterations. We can see here that feedback helps in slowly building up lines along proper orientations until we get almost all ridge lines (including those at the places where the underlying image is badly corrupted). The image in Fig. 5(a) is particularly bad because it has poor contrast, and hence, our line detector takes more time to stabilize. The line detector stabilizes in much fewer iterations on good images. 


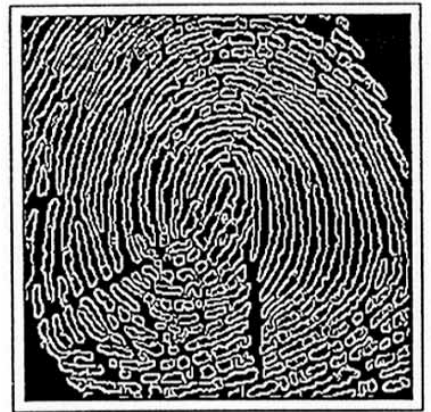

(a)

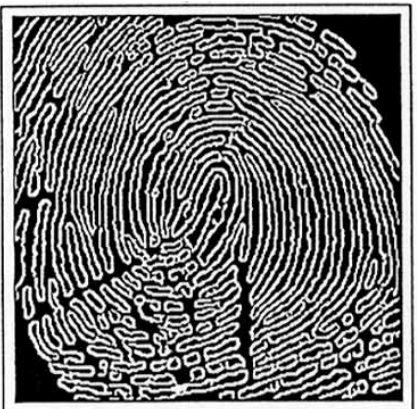

(c)

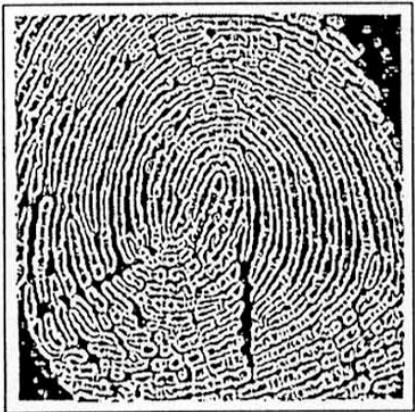

(e)

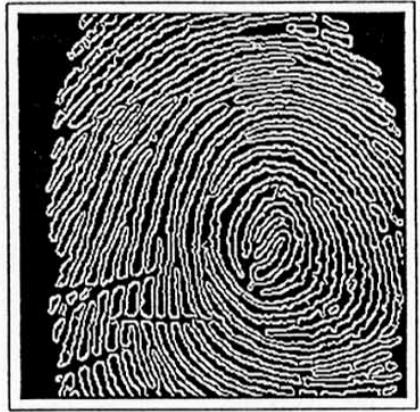

(b)

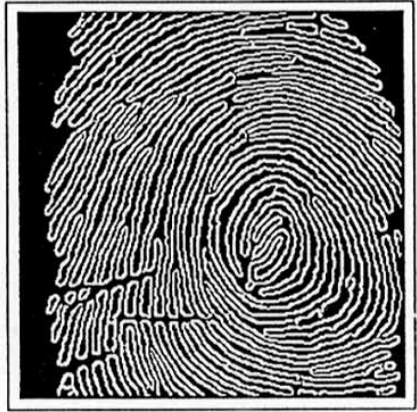

(d)

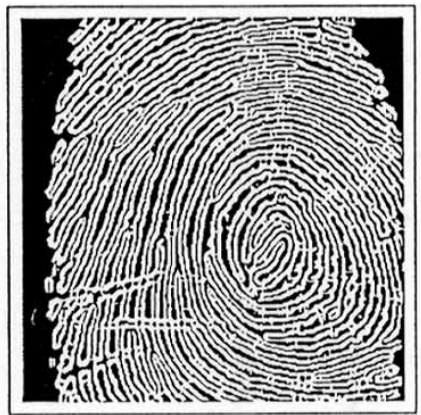

(f)
Fig. 4. Line outputs on the fingerprints in Fig. 3(a) using (a), (b) Canny with $\sigma=0.8$, (c), (d) Canny with $\sigma=1.5$, and (e), (f) logical/linear operator.

\section{B. Feature Extraction}

After obtaining the line output, we need to extract a low-dimensional feature vector to classify the fingerprint. The shape of orientation flow field, in terms of overall changes of orientations of ridge lines as we go around the fingerprint, carries information that is very useful for classifying the fingerprint. To characterize such information, we obtain two feature vectors $\mathbf{F} 1$ and $\mathbf{F} 2$ from the line detected fingerprint image. As explained in the next section, we tried three different classification strategies and get equally good performance. Some of our classifiers use $\mathbf{F}$ 1, whereas others use $\mathbf{F} 2$. In this subsection, we explain how the two feature vectors are obtained.

We obtain the first feature vector $\mathbf{F} 1$ as follows. We make a coarse approximation of orientation information in a fingerprint image by dividing the line detected image into blocks of size $16 \times 16$ and labeling every block by the major orientation in that $16 \times 16$ image. This gives us a reduced image where each

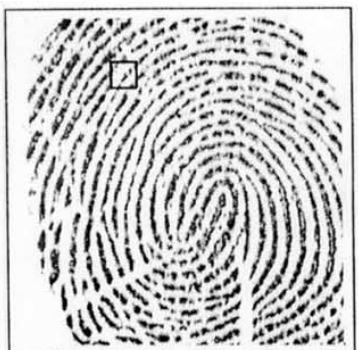

(a)

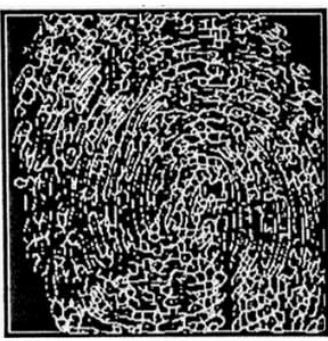

(c)

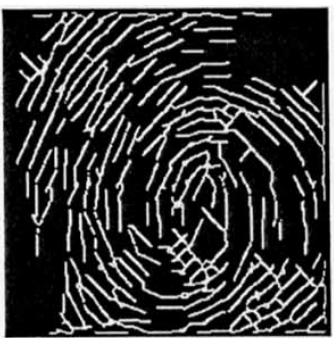

(e)

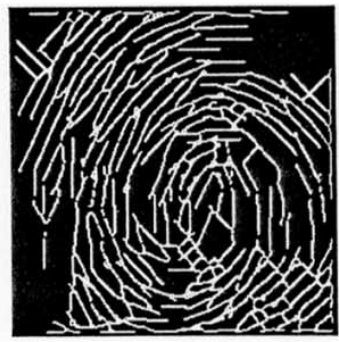

(g)

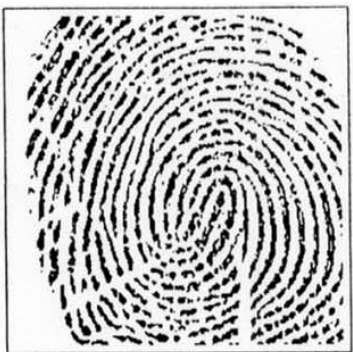

(b)

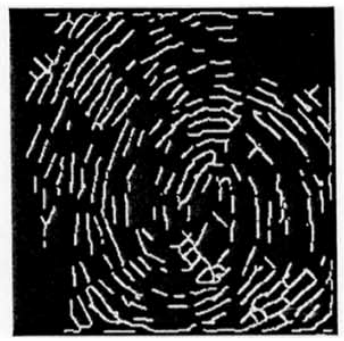

(d)

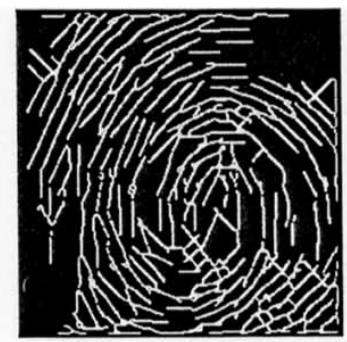

(f)

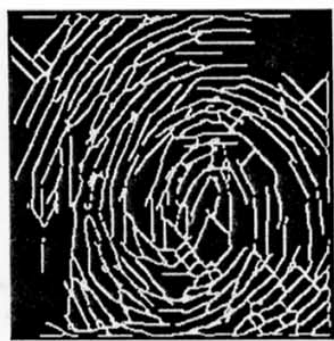

(h)

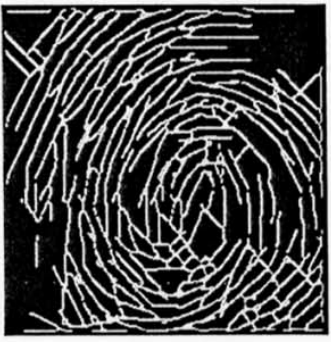

(i)

Fig. 5. Illustration of the utility of feedback. (a) Original image. (b) Binarized image. (c)-(i) Line outputs at $t=1,3,5,7,9,11$, and 15.

pixel value is an orientation. (If the original image is of size $512 \times 512$, then the reduced image will have size $32 \times 32$ ). This is illustrated in Fig. 6(a) by superposing the grid on the original image. To capture the flow distribution, the reduced image is 
partitioned into $24(=8 \times 3)$ zones, as shown in Fig. 6(b). (Here, the center point is chosen to be the center of the image array.) In every zone, we obtain the histogram of orientations. Then each zone is characterized by the two major orientations and their percentage of occurrence in the zone. Thus, we have a $96(=$ $24 \times 4$ )-dimensional feature vector for every fingerprint image. We call this feature vector $\mathbf{F} 1$.

In obtaining the above feature vector $\mathbf{F} 1$, the centerpoint used for dividing the reduced image into zones [see Fig. 6(b)] is arbitrary in the sense that it has nothing to do with the "center" of the fingerprint. We took it to be the center of the image. However, we can get better information about the orientation flow if we characterize the distribution of orientations around the true center of the fingerprint image. The second feature vector $\mathbf{F} 2$ is intended to do this.

To illustrate the usefulness of such a feature vector, Fig. 7 shows typical Arch and Tented Arch fingerprint images. As can be seen, these classes are very similar and differ from each other around the "center" region marked as a circle in the figure. To calculate F2, we find the "center" of fingerprint image as follows. Define the orientation entropy as

$$
O_{E}=\sum_{i=0}^{7} p_{i}\left(1-p_{i}\right)
$$

where $p_{i}$ is the fraction of ridge line pixels with orientation $i$.

On the line output of a fingerprint image, we find a subimage of size $120 \times 120$, say $S$, that has maximum entropy. ${ }^{3}$ Then, for $S$, we make a coarse approximation of orientation information by dividing it into blocks of size $15 \times 15$ pixels. For every block, we obtain histogram of orientations. Each block is characterized by the two major orientations in that block. This gives us a $128(=8 \times 8 \times 2)$-dimensional feature vector that represents $S$. We call this feature vector $\mathbf{F} 2$.

\section{Organization of Our Classifiers}

For the fingerprint classification problem, we have considered five ${ }^{4}$ classes: Arch (A), Left loop (L), Right loop (R), Tented arch (T), and Whorl (W). To do this multiclass classification, we used three different strategies, namely, SVMs, nearest-neighbor, and feed-forward neural networks.

The SVM is a recently developed technique [13], [14] for two-class pattern classification. It turns out that the problem of learning an SVM is that of optimization of a quadratic cost function with linear constraints for which many efficient numerical techniques exist [15], [19].

${ }^{3}$ To find $S$, we need to slide a $120 \times 120$ window on the image. For a $512 \times 512$ image, we need to consider about $16 \times 10^{4}$ windows. If this were a general mask operation with a $120 \times 120$ mask, then for each window, the number of operations would be of the order of $10^{4}$, making the overall computational cost of the order of $10^{8}$, which is prohibitive. However, in our case, in each window, we need to compute only $p_{i}, i=0,7$ and since we are sliding the window, we can obtain these values by looking at only $120+120$ pixels per window, making computation per window of the order of $10^{2}$. Thus, the computational cost of finding $S$ is not very high. To further reduce this cost, we actually slide the window by five pixels in each dimension.

${ }^{4}$ Since there are very few Scar fingerprints in the NIST database we worked with, we do not consider this class.

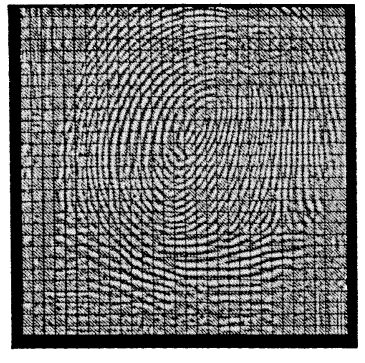

(a)

Fig. 6. Feature extraction (see text).

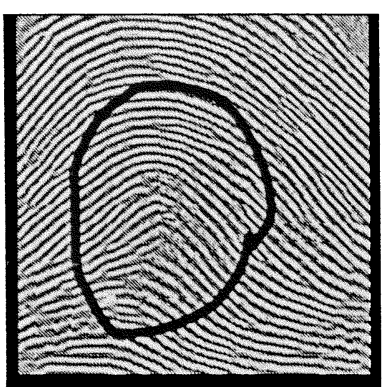

(a)

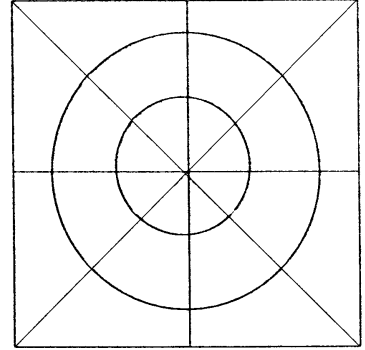

(b)

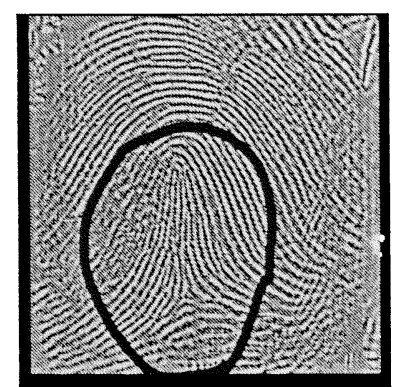

(b)
Fig. 7. Flow pattern characterizing (a) Class $\mathrm{A}$ and (b) Class $\mathrm{T}$, which motivated our second feature vector (see text).

One can adopt this two-class classification technique for multi-class problem with, say, $M$ classes by learning $M$ number of two-class classifiers, each separating one class from the rest. Thus, we need to learn five classifiers. For example, the first classifier can be trained to distinguish class-L against all others, and so on. With this kind of classifier (one against all), with more than two classes, we can classify a pattern based on the output from all classifiers. That is, the pattern is assigned to the class whose SVM has the highest positive output. If none of the SVMs have positive output, then we would reject such a pattern.

For our fingerprint classification, looking at the characteristics of various classes, we organized our SVMs as a hierarchical classifier as shown in Fig. 8. We first separate classes A and T from classes L, R, and W. For the next level of classification, we learn four classifiers: class A versus class $\mathrm{T}$, class $\mathrm{L}$ versus classes $\mathrm{R}$ and $\mathrm{W}$, class $\mathrm{R}$ versus classes $\mathrm{L}$ and $\mathrm{W}$, and class $\mathrm{W}$ versus classes $L$ and $R$. We used the features extracted from the line output of fingerprint image as explained in Section II-B. Fig. 8 also shows the details of feature vectors used by individual classifiers. Feature vector $\mathbf{F} 1$ is calculated for every fingerprint image. This feature vector is used by our top level SVM to decide whether the fingerprint is of class A,T or class L,R,W. At the second level, only the classifier that separates class A from class T uses F2. All others use feature vector $\mathbf{F} 1$. To learn each of the SVMs, we used the SMO algorithm [15] with parameters $C=1, \epsilon=0.001$, and $\gamma=0.0005$ and with Gaussian kernel function.

Our second method of fingerprint classification is by using nearest-neighbor classifier. Here, we need to select some prototype feature vectors and define a distance measure. To clas- 


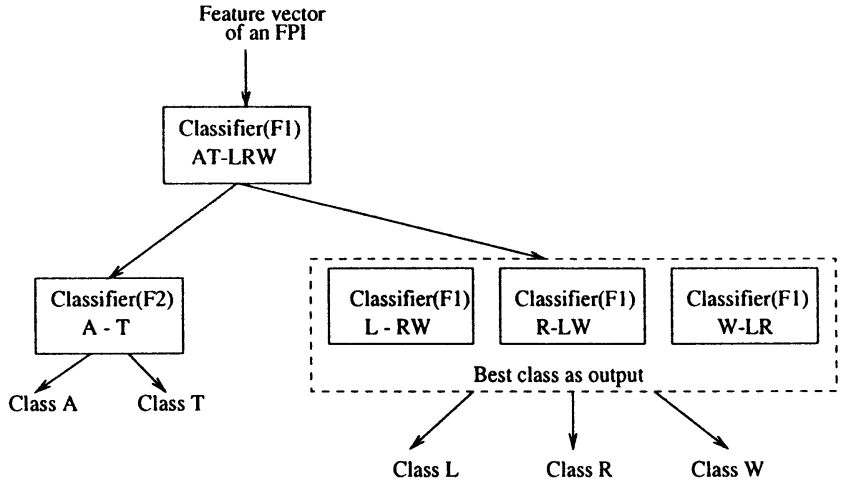

Fig. 8. Hierarchical fingerprint classifier: At each node, the feature vector used by each classifier is given in ( ).

sify a new fingerprint image, we measure the distance between every prototype and the new feature vector and then label it with the class of minimum distance prototype. For our five-class fingerprint classification problem, we used feature vector $\mathbf{F} 1$ and Euclidean distance. Efficiency and performance of nearestneighbor classifier is very much dependent on the prototypes. In the next section, we show that we can actually use the support vectors learned by SVMs as the prototypes which improves the accuracy and reduces the computational time of nearestneighbor classifier.

The third classifier we used here is based on feed-forward neural networks using the Backpropagation algorithm for training. To effect final classification, we employ the same hierarchical structure given in Fig. 8, but the classifier learned at each node is a feed-forward neural network. For Classifiers AT-LRW, L-RW, R-LW, and W-LR, we have 96 input nodes (because we use feature vector $\mathbf{F} 1$ for all these classifiers), three hidden nodes, and one output node. Classifier A-T has 128 input nodes (because we use feature vector F2) and two hidden layers: with three nodes in the first and two nodes in the second hidden layer and one output node. All our networks have only one output node because each of this is trained on a two-class classification problem. We take class label to be -1 or 1 . We have used the hyperbolic tangent function as the activation function for all the nodes in the network. Parameters of Backpropagation algorithms are learning rate $\eta=0.6$ and the momentum step size $\gamma=0.3$. We trained the network for 1500 epochs.

\section{Performance of Fingerprint Classifiers}

In this section, we present results obtained with our fingerprint classifiers on NIST database from pattern-level classification automation system (PCASYS) [3]. This database has 2700 gray-scale fingerprint images. There are only five images of Scar fingerprint in this database. So, we did not consider learning a classifier for this class. We classify a fingerprint image into one of the five classes: Arch (A), Left loop (L), Right loop (R), Tented arch (T), and Whorl (W). In the following subsections, we present the performance of our classifiers (described in Section II-C) and compare it with other published results.
TABLE I

ACCURACY OF LEARNED SVMS

\begin{tabular}{c||c|r|r|r|r}
\hline \multicolumn{1}{c||}{} & \multicolumn{2}{c|}{$\%$ Accuracy } & \multicolumn{3}{c}{ Number of Patterns } \\
\cline { 2 - 6 } Class & Train & Test & Train & Test & SV \\
\hline AT-LRW & 100 & 99.32 & 1590 & 1105 & 87 \\
A-T & 100 & 79.10 & 90 & 43 & 90 \\
L-RW & 100 & 100.00 & 1500 & 1062 & 58 \\
R-LW & 100 & 96.79 & 1500 & 1062 & 59 \\
W-LR & 100 & 94.07 & 1500 & 1062 & 58 \\
\hline
\end{tabular}

TABLE II

CONFUSION MATRIX WITH SVMS

\begin{tabular}{l||c|c|c|c||c|l}
\hline Class & L & R & W & AT & Accuracy & Patterns \\
\hline L & 801 & 0 & 0 & 3 & $99.6 \%$ & 804 \\
R & 0 & 688 & 0 & 16 & $93.6 \%$ & 735 \\
W & 0 & 2 & 998 & 0 & $97.6 \%$ & 1023 \\
AT & 2 & 0 & 0 & 129 & $97.0 \%$ & 133 \\
\hline
\end{tabular}

\section{A. Hierarchical Classifier Using Support Vector Machines}

To learn the hierarchical classifier, we use a subset of fingerprint images from the database as the training set. We test the performance with rest of the images. As shown in Fig. 8, our first classifier is for separating classes $\mathrm{A}$ and $\mathrm{T}$ from classes $\mathrm{L}$, $\mathrm{R}$, and $\mathrm{W}$. We used 1590 images as training set and 1105 images as test set. The classes L, R, and W together had 2562 images. We used 1500 of them as training set for learning the classifiers that separate each of the $\mathrm{L}, \mathrm{R}$, and $\mathrm{W}$ from the other two. The classes A and T together have only 133 images in the database. Hence, for the classifier that separates A from T, we used 90 images as the training set and 43 images as a test set. (All these details are given in Table I). In each case, the specific images that constitute the training set are randomly selected. It is observed that the classification accuracy is about the same with different random choices of training and test sets.

Table I summarizes the performance of five SVMs that we learned. For each SVM, the table shows the final accuracy obtained on the training set and test set as well as the number of patterns in the training set and test set. As can be seen, the classification accuracy is quite good. The last column of the table gives the number of support vectors found by the algorithm for each SVM. In an SVM, the final classification is determined by a subset of the training patterns called support vectors. If it so turns out that the number of support vectors is a small fraction of the number of training patterns, then we can have good confidence that the learned classifier generalizes well. ${ }^{5}$ As can be seen from Table I, for the three SVMs that recognize the classes $\mathrm{L}, \mathrm{R}$, and $\mathrm{W}$, only about 60 out of 1500 training patterns happen to be support vectors. For the SVM that separates classes A and $\mathrm{T}$ from L, R, and W, 87 out of 1590 training patterns happen to be support vectors. For all these SVMs, the accuracy obtained on test set is also very high. On the other hand, for the SVM that is required to distinguish between classes $\mathrm{A}$ and $\mathrm{T}$, all the training

\footnotetext{
${ }^{5}$ More precisely, the expected value of the probability of mis-classification is bounded above by the fraction of training patterns that turned out to be support vectors [14]
} 
patterns become support vectors and, as would be expected, the accuracy on test set is poor, even though accuracy on training set is $100 \%$. We feel that the main problem here is that the number of training patterns available is small. Given enough training patterns, the SVM method with our feature vector should deliver good performance for class A versus $\mathrm{T}$ as well.

The final classification accuracy is presented in Table II in the form of a confusion matrix. For an $M$-class classification problem, the confusion matrix will be an $M \times M$ matrix. The $(i, j)$ th element of the confusion matrix shows the number of patterns from class $i$ in the database that are classified as class $j$ by the classifier. Thus, for an ideal classifier all off diagonal elements in the confusion matrix would be zero. Since the discrimination obtained between $\mathrm{A}$ and $\mathrm{T}$ is poor (as shown in Table I), in the confusion matrix, we clubbed the classes $\mathrm{A}$ and $\mathrm{T}$ together. Hence, in the confusion matrix, we have only four classes: L, $\mathrm{R}, \mathrm{W}$, and AT. From Table II, it is seen that the final classification accuracy obtained is very good. The last column of the table gives the number patterns of each class in the database. It may be noted that the confusion matrix accounts for only 2639 patterns. The remaining 56 patterns were not classified because our hierarchical classifier rejects them. That is, while classifying a pattern at first level based on the sign of SVM output, we would follow either the left or right branch. At the second level, when a pattern is from either class $\mathrm{L}, \mathrm{R}$, or $\mathrm{W}$, if none of the three SVMs have positive output, then we would have to reject such a pattern. However, as can be seen from Table II (e.g., row W), for calculating the accuracy of the classifier, we have taken all rejects as errors.

Since learning any SVM classifier involves solving only a quadratic programming problem, the method is very efficient. With 1500 training patterns, learning of each of the SVMs took about $2.2 \mathrm{~s}$ on a Pentium $300 \mathrm{MHz}$ PC. (This time does not include that for line detection and feature extraction on all the 1500 images, which took about 13 s/image.) We have used the SMO algorithm [15] for learning SVMs. It is observed that the final classification accuracies are very robust with respect to choice of parameters in the SMO algorithm. We have used the same parameters values for all SVMs. There are algorithms for learning SVMs, which are reported to be four to five times faster than SMO [19]. After learning all SVMs, using our hierarchical classifier, the time needed for classifying a new fingerprint image is $13.1 \mathrm{~s}$. (This time includes time needed for line detection and feature extraction.)

\section{B. Nearest-Neighbor Classifier}

We also implemented a nearest-neighbor classifier with Euclidean distance on feature vector $\mathbf{F} 1$. As prototypes, we have taken 500 fingerprints for each of class L, R, and W, $50 \mathrm{im}-$ ages of class A, and 40 images of class T. Thus, there are 1590 prototypes (same as the number of training samples used for SVM). Once again, the specific images to be prototypes are selected randomly. Table III shows the confusion matrix for a nearest-neighbor classifier with 1590 prototypes. The performance is good, although we can see that our SVM-based hierarchical classifier performs better specially for class AT.

To label a new fingerprint image with one of the five classes, the nearest-neighbor classifier needs to compute the distance
TABLE III

CONFUSION MATRIX FOR NEAREST NEIGHBOR ClASSIFIER WITH 1590 PROTOTYPES

\begin{tabular}{l||c|c|c|c||r}
\hline Class & L & R & W & AT & $\%$ Accuracy \\
\hline L & 804 & 0 & 0 & 0 & 100.00 \\
R & 0 & 735 & 0 & 0 & 100.00 \\
W & 6 & 7 & 1009 & 1 & 98.63 \\
AT & 3 & 7 & 0 & 123 & 92.48 \\
\hline
\end{tabular}

TABLE IV

CONFUSION Matrix FOR NEAREST NeIGHBor Classifier With 100 RANDOMLY CHOSEN PROTOTYPES

\begin{tabular}{c||c|c|c|c||r}
\hline Class & L & R & W & AT & \%Accuracy \\
\hline L & 732 & 0 & 0 & 72 & 91.04 \\
R & 0 & 678 & 0 & 57 & 92.20 \\
W & 38 & 93 & 892 & 0 & 87.20 \\
AT & 14 & 33 & 17 & 69 & 51.90 \\
\hline
\end{tabular}

TABLE $\mathrm{V}$

CONFUSION MATRIX FOR $N N_{\text {svm }}$ ClASSIFIER

\begin{tabular}{c||c|c|c|c||r}
\hline Class & L & R & W & AT & \%Accuracy \\
\hline L & 804 & 0 & 0 & 0 & 100.00 \\
R & 0 & 745 & 0 & 0 & 100.00 \\
W & 3 & 8 & 1011 & 1 & 98.83 \\
AT & 3 & 4 & 0 & 126 & 94.70 \\
\hline
\end{tabular}

between the new feature vector and all the prototypes. With 1590 prototypes, this takes a lot of time. We can reduce the time taken by reducing the number of prototypes, but this may affect the accuracy. In Table IV, we show accuracy obtained with a nearest-neighbor classifier if we choose 100 randomly selected images as prototypes. As can be see, the accuracy is poor. This is because the random prototypes chosen may not be very representative. Deciding which training patterns would be good as prototypes is, in general, difficult. An interesting method for choosing prototypes could be to use the learned SVMs itself. The support vectors in the SVM are, essentially, the most critical patterns for the particular classification problem. In our hierarchical classifier of SVMs, for all the SVMs together, we had about 150 distinct patterns as support vectors. We can use the support vectors as prototypes for the nearest-neighbor classifier. We call this nearest-neighbor classifier with support vectors as prototypes by $N N_{\mathrm{svm}}$. The confusion matrix in Table $\mathrm{V}$ summarizes the results with the $N N_{\text {svm }}$ classifier. Performance of the $N N_{\text {svm }}$ classifier is best both in terms of efficiency and accuracy.

\section{Hierarchical Classifier With Feed-Forward Neural Networks}

We also implemented a hierarchical classifier of feed-forward neural networks (as given in Section II-C). We first train the neural networks with training set (which is the same as that used by SVMs) and obtain weight matrix for each neural network. Then, we do classification by following the hierarchical structure (see Fig. 8) based on outputs of neural network at each node. 
TABLE VI

ACCURACY OF LEARNED FEED ForWARD NEURAL NETWORKS

\begin{tabular}{c||c|r|r|r}
\hline \multicolumn{1}{c||}{} & \multicolumn{2}{c|}{$\%$ Accuracy } & \multicolumn{2}{c}{ Number of Patterns } \\
\cline { 2 - 5 } Class & Train & Test & Train & Test \\
\hline AT-LRW & 100 & 99.46 & 1590 & 1105 \\
A-T & 100 & 81.00 & 90 & 43 \\
L-RW & 100 & 100.00 & 1500 & 1062 \\
R-LW & 100 & 100.00 & 1500 & 1062 \\
W-LR & 100 & 97.92 & 1500 & 1062 \\
\hline
\end{tabular}

TABLE VII

CONFusion Matrix With FEED-Forward NeURAL NeTworks

\begin{tabular}{l||c|c|c|c||r|r}
\hline Class & L & R & W & AT & Accuracy & Patterns \\
\hline L & 798 & 0 & 0 & 6 & $99.2 \%$ & 804 \\
R & 0 & 735 & 0 & 0 & $100.0 \%$ & 735 \\
W & 0 & 7 & 979 & 0 & $95.7 \%$ & 1023 \\
AT & 2 & 3 & 0 & 128 & $96.2 \%$ & 133 \\
\hline
\end{tabular}

Table VI gives the classification accuracies. As can be seen, this classifier also performs well. From Table VII, it is seen that the final classification accuracy obtained by this classifier is good. It may be noted that 37 patterns were rejected by this hierarchical classifier. Hence, the confusion matrix has only 2658 patterns.

\section{Other Fingerprint Classifiers}

We compare our results with the PCASYS system [3], which uses exactly the same database that we used. In PCASYS, to classify a fingerprint the program performs these processing steps: image enhancement (filtering); feature extraction (local ridge orientations); registration (use of a linear transform, which applies a pattern of regional weights and reduces the dimensionality); running a probabilistic neural network (PNN) classifier and of an auxiliary whorl-detector that traces and analyzes pseudo ridges (approximate trajectories through the ridge flow). Finally, the outputs of the PNN and the pseudo ridgetracer are combined to produce a class output. Table VIII gives the confusion matrix obtained by PCASYS system. Comparing Table VIII with the results of Tables II, V, and VII, we indeed perform better.

We also compare our fingerprint classification results with those published in [8]-[10] with various other fingerprint databases from NIST. The fingerprint classification algorithm presented by Jain et al. [8], classifies fingerprints into five categories using a two-stage classifier. On the NIST-4 database (with 4000 patterns), for a five-class fingerprint classification problem, they achieved $90 \%$ classification accuracy (with $1.8 \%$ reject at feature extraction stage). Their classification accuracy was further increased to $96 \%$ after a total of $32.5 \%$ images were rejected. Watson et al. in [9] used two types of Fourier transform-based filters to enhance fingerprint images and used neural network for fingerprint classification. Classification tests were performed with fingerprints from NIST special database 9 , volume $1-5$, using the ridge-valley based feature vector and the PNN classifier. They were able to achieve
TABLE VIII

CONFUSION MATRIX FOR PCASYS SYSTEM

\begin{tabular}{l||c|c|c|c|c}
\hline Class & L & R & W & AT & \%Accuracy \\
\hline L & 779 & 2 & 13 & 10 & 96.9 \\
R & 11 & 696 & 20 & 8 & 94.7 \\
W & 45 & 28 & 950 & 0 & 92.7 \\
AT & 26 & 17 & 0 & 90 & 67.7 \\
\hline
\end{tabular}

TABLE IX

Classification ACCURACY REPORTED WITH 10\% REJECTS

\begin{tabular}{l||r|r}
\hline Classifier & \% Accuracy & No. of features \\
\hline MLP & 91.8 & 64 \\
RBF & 91.9 & 64 \\
PNN & 92.8 & 112 \\
\hline
\end{tabular}

$91.35 \%$ classification accuracy with $10 \%$ rejects. Blue et al. [10] have evaluated classification accuracy of various statistical and neural network classifiers for fingerprint classification. Their classifiers were trained and tested using images from the NIST SD-4 database with 4000 images. They were able to achieve $93 \%$ classification accuracy with $10 \%$ rejects. Table IX summarizes results from [10]. Comparing our fingerprint classification accuracies with Table IX, we can say that our classifiers achieve good performance.

\section{CONCLUSION}

In this paper, we presented an algorithm for classifying fingerprint images into five classes, namely, Arch, Tented arch, Left loop, Right loop, and Whorl. After binarizing the fingerprint image using an adaptive threshold, we do line detection using a novel oriented line detector. We then extract low-dimensional feature vectors that essentially characterizes the variation in the orientation of ridge lines around the fingerprint. For the classification, we used three different classifiers. As discussed in Section III, all our classifiers deliver good classification accuracy. We also compared our classification results with other published work. Our classifiers give better accuracies, and they are also very efficient. Since all our classification strategies achieve good accuracy, we feel that our novel line detection algorithm and feature extraction process are quite effective in capturing the information from a fingerprint image that is crucial for classification. An interesting aspect of our results is the performance achieved by $N N_{\text {svm }}$. This illustrates the effectiveness of the SVM method in identifying the "critical" patterns in a training set.

As can be seen from Figs. 2 and 3, our feedback-based line detector does a good job of identifying clean ridge lines with proper orientation information. The line detector given here is a new specialized version of a general feedback based line detector discussed in [11]. The line detection algorithm in [11] is motivated by some aspects of feedback connections in the early visual pathway in mammalian brain. It uses differentiated oblong Gaussian as simple cell edge masks, has a specially constructed receptive field as a mask in obtaining outputs of line 
cells from simple cells, and has two levels of feedback. The algorithm presented here is like any other simple oriented edge detection for binary images except for incorporation of one feedback mechanism. As illustrated in Fig. 5, the iterative refinement of lines using feedback is very effective for fingerprint images. The line detector works on binary images. Our binarization method using an adaptive threshold works well for all images from the NIST database [3]. However, for more noisy fingerprint images, we may need better binarization technique. The line detection presented should also be useful for other applications such as fingerprint-based recognition. Since our line detector gives the orientation of lines at every point and it preserves points where lines with different orientations meet, it should be useful for, e.g., minutiae detection. This aspect of our line detector would be investigated in our future work.

\section{REFERENCES}

[1] A. K. Jain, L. Hong, and S. P. Bolle, "An identity-authentication system using fingerprints," Proc. IEEE, vol. 85, pp. 1365-1388, Sept. 1997.

[2] A. R. Roddy and J. D. Stosz, "Fingerprint features-Statistical analysis and system performance estimates," Proc. IEEE, vol. 85, pp. 1390-1421, Sept. 1997.

[3] "PCASYS-A Pattern-level classification automation system for fingerprints," Visual Image Processing Group, National Inst. Stand. Technol., Boulder, CO, Tech. Rep. NISTIR 5647, 1995.

[4] M. Kawagoe and A. Tojo, "Fingerprint pattern classification," Pattern Recogn., vol. 17, no. 3, pp. 295-303, 1984.

[5] A. P. Fitz and R. J. Green, "Fingerprint calssification using a hexagonal fast Fourier transform," Pattern Recogn., vol. 29, no. 10, pp. 1587-1597, 1996.

[6] K. Karu and A. K. Jain, "Fingerprint classification," Pattern Recogn., vol. 29, no. 3, pp. 389-404, 1996.

[7] M. M. Chong, T. H. Ngee, L. Jun, and R. K. Gay, "Geometric framework for fingerprint image classification," Pattern Recogn., vol. 30, no. 9, pp. 1475-1488, 1997.

[8] A. K. Jain, S. Prabhakar, and L. Hong, "A multichannel approach to fingerprint classification," IEEE Trans. Pattern Anal. Machine Intell., vol. 21, pp. 348-359, Apr. 1999.

[9] C. L. Watson, G. T. Candela, and P. J. Grother, Comparison of FFT Fingerprint Filtering Methods for Neural Network Classification, Sept. 1994.

[10] J. L. Blue, G. T. Candela, P. J. Grother, R. Chellappa, C. L. Wilson, and J. D. Blue, "Evaluation of pattern classifiers for fingerprint and OCR applications," Pattern Recogn., vol. 27, pp. 485-501, 1994.

[11] P. S. Sastry, S. Shah, S. Singh, and K. P. Unnikrishnan, "Role of feedback in mammalian vision: A new hypothesis and a computational model," Vision Res., vol. 39, pp. 131-148, Jan. 1999.

[12] S. Shah, P. S. Sastry, and K. P. Unnikrishnan, "A feedback based algorithm for line detection," in Proc. Indian Conf. Comput. Vision, Graphics Image Process., Delhi, India, Dec. 1998.
[13] C. Burges, "A tutorial on support vector machine for pattern recognition," Data Mining Knowledge Discovery, vol. 2, pp. 1-47, 1998.

[14] V. Vapnik, "An overview of statistical learning theory," IEEE Trans. Neural Networks, vol. 10, pp. 988-999, Sept. 1999.

[15] J. Platt, "Sequential minimal optimization: A fast algorithm for training support vector machines," in Advances in Kernel Methods: Support Vector Learning, B. Scholkopf, C. J. C. Burges, and A. Smola, Eds. Cambridge, MA: MIT Press, 1999, pp. 185-208.

[16] E. Davies and A. P. Plummer, "Thinning algorithms: A critique and a new methodology," Pattern Recogn., no. 14, pp. 53-63, 1981.

[17] J. Canny, "A computational approach to edge detection," IEEE Trans. Pattern Anal. Machine Intell., vol. 8, pp. 679-698, June 1986.

[18] L. A. Iverson and S. W. Zucker, "Logical linear operators for image curves," IEEE Trans. Pattern Anal. Machine Intell., vol. 17, pp. 982-996, June 1995.

[19] C. Bhattacharyya, S. S. Keerthi, S. Shevade, and K. R. K. Murthy, "A fast iterative nearest point algorithm for support vector machine classifier design,” IEEE Trans. Neural Networks, vol. 11, pp. 124-136, Jan. 2000.

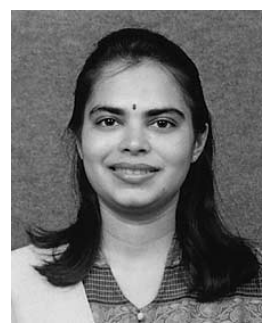

Shesha Shah received the B.E. degree in computer science and engineering from the M.S. University of Baroda, Baroda, India, in 1992 and the M.Sc.(Engg.) and $\mathrm{Ph} . \mathrm{D}$. degrees from the Department of Electrical Engineering, Indian Institute of Science, Bangalore, in 1996 and 2002, respectively.

She is currently working as a consultant for Strand Genomics, Bangalore, India. Her research intersts include pattern recognition, computational neuroscience, image processing, and bio-informatics.

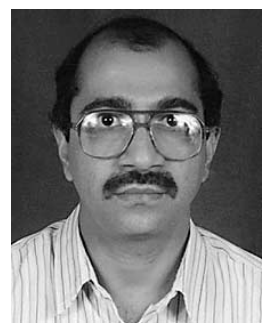

P. S. Sastry (S'82-M'85-SM'97) received B.Sc. (Hons.) degree in physics from Indian Institute of Technology, Kharagpur, in 1978 and the B.E. degree in electrical communications engineering and the Ph.D. degree in electrical engineering from the Indian Institute of Science (IISc), Bangalore, in 1981 and 1985, respectively.

Since 1986 he has been with the Faculty of the Department of Electrical Engineering, Indian Institute of Science, Bangalore, where currently he is a Professor. He has held visiting positions at the University of Massachusetts, Amherst; University of Michigan, Ann Arbor; and General Motors Research Labs, Warren, MI. His research interests include learning systems, pattern recognition, image processing, and computational neuroscience.

Dr. Sastry is a reciepient of the Alumni Medal for best thesis from the Division of Electrical Sciences, IISc in 1985, the Indo-US Science and Technology Fellowship in 1992, and the Sir C. V. Raman award in Computer Science in 1999. 